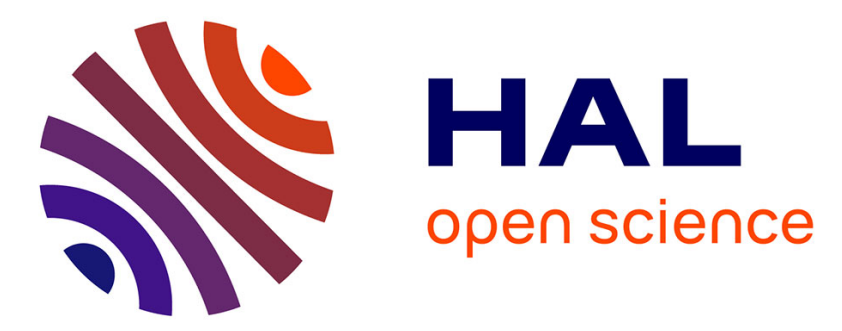

\title{
Random tessellations and Boolean random functions
}

\author{
Dominique Jeulin
}

\section{To cite this version:}

Dominique Jeulin. Random tessellations and Boolean random functions. 11th International Symposium, ISMM 2013, May 2013, Uppsala, Sweden. pp.25-36, 10.1007/978-3-642-38294-9_3 . hal$00739932 \mathrm{v} 2$

\section{HAL Id: hal-00739932 \\ https://hal-mines-paristech.archives-ouvertes.fr/hal-00739932v2}

Submitted on 1 Mar 2013

HAL is a multi-disciplinary open access archive for the deposit and dissemination of scientific research documents, whether they are published or not. The documents may come from teaching and research institutions in France or abroad, or from public or private research centers.
L'archive ouverte pluridisciplinaire HAL, est destinée au dépôt et à la diffusion de documents scientifiques de niveau recherche, publiés ou non, émanant des établissements d'enseignement et de recherche français ou étrangers, des laboratoires publics ou privés. 


\title{
Random tessellations and Boolean random functions
}

\author{
Dominique Jeulin \\ Centre de Morphologie Mathématique, \\ Mathématiques et Systémes, 35 rue Saint-Honoré, 77300 Fontainebleau, France
}

\begin{abstract}
Generalizations of various random tessellation models generated by Poisson point processes are proposed and their functional probability $P(K)$ is given. They are interpreted as characteristics of Boolean random functions models, which provide a generic way of simulation of general random tessellations.
\end{abstract}

Key words: Voronoi tessellation, random tessellation, Boolean random function

\section{Introduction}

Some models of random tessellations in the Euclidean space $R^{n}$ are defined from distances to the points $x_{k}$ of a point process, usually the Poisson point process $\mathcal{P}$ : the Voronoi tessellation is defined from the zones of influence of points $x_{k}$. Its generalizations like the Johnson-Mehl and the Laguerre tessellations use a time sequence of points, and a sequence of ponderations allocated to each point. It turns out that these models can be re-interpreted in the framework of Boolean random functions, with appropriate primary functions. In what follows, we propose new models of random tessellations based on local metrics attached to each point of the process. They correspond to specific Boolean random function models, which can be used for their simulation. The new models show a wide flexibility, generating tessellations with non planar boundaries, that can be used to simulate metallic grains [1] or foams [10].

\section{Reminder on random tessellations}

Random tessellations were formalized by G. Matheron in [11].

Definition 1. Consider a locally compact denumberable space $E$ and subsets $C_{i}$ of $E$, belonging to $\wp(E)$. A tessellation $\Theta$ is a collection of classes $C_{i} \in \wp(E)$ with

$$
\cup_{i} C_{i}=E \text { and } C_{i} \cap C_{j}=\emptyset \text { for } i \neq j
$$

We note $\Pi(E)$ (namely $\Pi_{g}(E)$ ) the set of tessellations of $E$ (namely of tessellations with open (or point) classes). $R_{A}$ is the subset of $\Pi_{g}(E)$, such that $A \subset E$ is contained in one class $C$. 
Subsets $R_{G}$, where $G$ are open parts of $E$, generate a $\sigma$ algebra on $\Pi_{g}(E)$, $\sigma\left(R_{G}\right)$, on which a probability can be constructed. A random tessellation $\Theta$ is characterized by $P\left(R_{G}\right)=P\left\{G \subset C_{i}\right\}$. In the present paper, we study some models of random tessellations in the Euclidean space $R^{n}$. In addition, we will consider locally finite random tessellations, for which the random number of classes in every bounded domain $D$ is a finite random number $N(D)$.

With this $\sigma$ algebra, we can define events (and their probability) like " $x$ belongs to a single class", " $x_{1}$ and $x_{2}$ " belong to a single class, " $x_{1}, x_{2}, \ldots x_{m}$ belong to $k$ classes, or more generally "the compact set $K$ is included in a single class". Note that the classes of a random tessellation in $R^{n}$ can be split in several connected components, as is the case for the dead leaves tessellation [6], or for some tessellations introduced in this paper.

\section{$3 \quad L_{p}$ Voronoi tessellations}

\subsection{Standard Voronoï tessellation}

Definition 2. The Poisson Voronoï tessellation in the Euclidean space $R^{n}$ is defined from zones of influence of Poisson points [4,16, 17]. The class $C_{k}$ of the tessellation containing point $x_{k}$ of the Poisson point process $\mathcal{P}$ is defined by

$$
C_{k}=\left\{x \in R^{n}, d\left(x, x_{k}\right)<d\left(x, x_{l}\right), x_{k} \in \mathcal{P}, x_{l} \in \mathcal{P}, l \neq k\right\}
$$

It is easy to show that every cell of the tessellation is an open set. Its closure is delimited by planar faces (planes in $R^{3}$ and segments in $R^{2}$ ) orthogonal to segments connecting neighbour points of $\mathcal{P}$. Indeed, using the Euclidean distance with

$$
d^{2}\left(x, x_{k}\right)=\sum_{i=1}^{i=n}\left(x_{i}-x_{k i}\right)^{2}
$$

$x_{i}$ being the coordinates of point $x$ in $R^{n}$, the boundary separating cells $C_{k}$ and $C_{l}$ is obtained by means of

$$
d^{2}\left(x, x_{k}\right)=d^{2}\left(x, x_{l}\right),
$$

generating a linear equation with respects to coordinates $x_{i}$, which provides the equation of an hyperplane.

The physical interpretation of this model is the isotropic growth from random point germs. Two-phase models of materials were generated from 3D Voronoi tessellations in [7].

\subsection{Anisotropic Voronoï tessellation}

A first change of the model is obtained by a non isotropic growth of germs. This can be made by using a Euclidean metric with positive eigenvalues $\lambda_{i}$ and 
with orthogonal eigenvectors obtained by a rotation of the basis of vectors $e_{i}$. In that case, the Euclidean metric is represented by a symmetric positive definite matrix $M(n, n)$. Noting $X$ and $X_{k}$ the vectors with coordinates $x_{i}$ and $x_{k i}$, we get $d^{2}\left(x, x_{k}\right)=\left(X^{t}-X_{k}^{t}\right) M\left(X-X_{k}\right)$, noting $X^{t}$ the transpose of vector $X$. Changing the Euclidean metric is equivalent to performing affine transformations in the directions of the eigenvectors, with ratios $\lambda_{i}$. Therefore, the resulting Voronoï tessellation is obtained by performing the corresponding affine transformations to the standard Voronoï tessellation, resulting in an anisotropic model, as considered in [15].

\subsection{Use of the $L_{p}$ metric}

Replacing the Euclidean metric by the $L_{p}$ metric produces new models of tessellations. We have for the $L_{p}$ metric with the integer $p$

$$
d^{p}\left(x, x_{k}\right)=\sum_{i=1}^{i=n}\left|x_{i}-x_{k i}\right|^{p}
$$

The separation between cells becomes

$$
d^{p}\left(x, x_{k}\right)=d^{p}\left(x, x_{l}\right) .
$$

When $p>1$, this expression gives polynomials with degree $p-1$ with respect to coordinates. For $p=\{1,2\}$ the separations are planar. For $p=3$, we get portions of quadrics. Increasing the value of $p$ gives higher order polynomial surfaces. However the obtained tessellations are not isotropic in the Euclidean space, since the balls defined by $\sum_{i=1}^{i=n}\left|x_{i}\right|^{p}=r^{p}$ are not isotropic, except for $p=2$, giving spheres. For $p=1$, the balls are hypercubes with edges orthogonal to directions given by $( \pm 1, \pm 1, \ldots, \pm 1)$. When $p \rightarrow \infty$ we get the $L_{\infty}$ metric, for which balls are hypercubes with edges parallel to the coordinates system. For $p=1$ and $p=\infty$, the separations are parallel to the faces of the corresponding hypercubes.

\subsection{Tesselations defined from local metrics}

To simulate locally anisotropic growth and local growth rates, it is interesting to start with a field of metrics depending on the location $x$. Depending on variations of the metric in space, tessellations with oriented cells following a field of orientations will be produced. This approach is followed by [8] in the context of meshing, using local Euclidean metrics, but no probabilistic properties is given.

Tesselations defined with a local Euclidean metric We will now attach to every Poisson point $x_{k}$ the Euclidean metric defined by the matrix $M_{k}$. In general the matrices corresponding to different germs will be correlated.

The definition of the tessellation (2) becomes: 
Definition 3. The local Poisson Voronoï tessellation in the Euclidean space $R^{n}$ is defined from zones of influence of Poisson points, using the Euclidean distance $d_{k}$ for point $x_{k}$. The class $C_{k}$ of the tessellation containing point $x_{k}$ is defined by

$$
C_{k}=\left\{x \in R^{n}, d_{k}\left(x, x_{k}\right)<d_{l}\left(x, x_{l}\right), x_{k} \in \mathcal{P}, x_{l} \in \mathcal{P}, l \neq k\right\}
$$

The separation between cells $C_{k}$ and $C_{l}$ is given by the equation

$$
\left(X^{t}-X_{k}^{t}\right) M_{k}\left(X-X_{k}\right)=\left(X^{t}-X_{l}^{t}\right) M_{l}\left(X-X_{l}\right)
$$

Rearranging the terms in equation (5), we get

$$
X^{t}\left(M_{k}-M_{l}\right) X-2 X^{t}\left(M_{k} X_{k}-M_{l} X_{l}\right)+X_{k}^{t} M_{k} X_{k}-X_{l}^{t} M_{l} X_{l}=0
$$

The separations of cells are made of portions of quadrics, and the edges are therefore portions of conics. Note that the cells $C_{k}$ can be made of several connected components.

Tesselations defined with a local $\boldsymbol{L}_{p}$ metric We will now consider attached to each germ $x_{k}$ a local $L_{p}$ metric defined on a basis obtained from the orthonormal basis of $R^{n}$ by a rotation matrix $R_{k}$ and a system of positive weights $a_{k i}$. In this basis, the coordinates of point $x$ become $X^{\prime}=R X$ and expression (3) becomes

$$
d_{k}^{p}\left(x, x_{k}\right)=\sum_{i=1}^{i=n} a_{k i}\left|x_{i}^{\prime}-x_{k i}^{\prime}\right|^{p}
$$

The definition of the tessellation (2) becomes:

Definition 4. The local Poisson Voronoï tessellation in the Euclidean space $R^{n}$ is defined from zones of influence of Poisson points, using the $L_{p}$ metric $d_{k}$ for point $x_{k}$. The class $C_{k}$ of the tessellation containing point $x_{k}$ is defined by

$$
C_{k}=\left\{x \in R^{n}, d_{k}^{p}\left(x, x_{k}\right)<d_{l}^{p}\left(x, x_{l}\right), x_{k} \in \mathcal{P}, x_{l} \in \mathcal{P}, l \neq k\right\}
$$

As before, cells $C_{k}$ are not necessarily connected. Their separations are made of portions of hypersurfaces of degree $p$, and the edges are portions of curves of degree $p$.

\subsection{Calculation of the probability $P(K)$}

Voronoï tessellation with a constant metric $\boldsymbol{L}_{\boldsymbol{p}}$ Consider a compact set

$K$. General expressions of the probability $P(K)=P\left(K \subset C_{k}\right)$ can be derived for the Voronoi models defined from a Poisson point process with intensity $\theta(x)$, by generalization of the results of Gilbert [4]. We note $B(x, r)$ the ball with center $x$ and radius $r$, defined from the metric $L_{p}$. We have $B(x, r)=\left\{y, d^{p}(x, y) \leq r^{p}\right\}$ 
Theorem 1. Consider a Voronoi tessellation of space defined from the Poisson point process with intensity $\theta(x)$, and the metric $L_{p}$. The probability $P(K)=$ $P\left(K \subset C_{k}\right)$ is given by

$$
P(K)=\int_{R^{n}} \theta(d y) \exp -\theta(F(K, y))
$$

where $\theta(F(K, y))=\int_{R^{n}} \theta(d x) 1_{F(K, y)}(x)$ is the measure of the Voronoi flower $F(K, y)=\cup_{x \in K} B(x, d(x, y))$. In the stationary case, for a constant intensity $\theta$, equation (9) becomes

$$
P(K)=\theta \int_{R^{n}} \exp -\theta \mu_{n}(F(K, y)) d y,
$$

$\mu_{n}$ being the Lebesgue measure in $R^{n}$.

Proof. We have $K \subset C_{k} \Longleftrightarrow \forall x \in K, \forall l \neq k, d\left(x, x_{k}\right) \leq d\left(x, x_{l}\right) \Leftrightarrow$ the ball with center $x \in K$ and radius $d\left(x, x_{k}\right)$ contains no point of the Poisson point process. Calling $F(K, y)=\cup_{x \in K} B(x, d(x, y))$ the Voronoi flower [2] of $K$ with center $y$, we have $K \subset C_{k} \Leftrightarrow F\left(K, x_{k}\right)$ contains no point of the process, with probability $\exp -\theta\left(F\left(K, x_{k}\right)\right)$. Equation (9) is obtained by randomization of the point $x_{k}, \theta(d y)$ being the probability that the element of volume $d y$ contains a point of the process.

When $K$ is a connected compact set, $P(K)$ gives the probability for $K$ to be included in a single connected component of the cell.

Tesselations defined with a local $\boldsymbol{L}_{p}$ metric We consider now random tessellations with local $L_{p}$ metrics $d_{k}^{p}$ and $d_{l}^{p}$, attached to germs $x_{k}$ and $x_{l}$. These random metrics, defined by a set of random coefficients and a rotation, are independent for separate germs. They are characterized by some multivariate distribution function noted $\varphi(k)$. We note $B_{l}(x, r)$ the ball with center $x$ and radius $r$, defined from the metric $d_{l}^{p}$. We have $B_{l}(x, r)=\left\{y, d_{l}^{p}(x, y) \leq r^{p}\right\}$. We call $F_{l k}(K, y)=\cup_{x \in K} B_{l}\left(x, d_{k}(x, y)\right)$ the flower of $K$ with center $y$, and metrics $d_{l}^{p}$ and $d_{k}^{p}$.

Theorem 2. Consider a random tessellation of space with local $L_{p}$ metrics, defined from the Poisson point process with intensity $\theta(x)$. The probability $P(K)=$ $P\left(K \subset C_{k}\right)$ is given by

$$
P(K)=\int_{R^{n}} \varphi(l) d l \varphi(k) d k \theta(d y) \exp -\theta\left(F_{l k}(K, y)\right)
$$

In the stationary case, for a constant intensity $\theta$, equation (11) becomes

$$
P(K)=\theta \int_{R^{n}} \varphi(l) d l \varphi(k) d k \exp -\left(\theta \mu_{n}\left(F_{l k}(K, y)\right)\right) d y
$$


Proof. Conditionally to the metrics $d_{k}^{p}$ and $d_{l}^{p}$, and to the location of a Poisson point $x_{k}$, we have $K \subset C_{k} \Longleftrightarrow \forall x \in K, \forall l \neq k, d_{k}\left(x, x_{k}\right) \leq d_{l}\left(x, x_{l}\right)$. Therefore, for every point $x$ of $K$, the ball $B_{l}\left(x, d_{k}\left(x, x_{k}\right)\right)$ contains no point of the process, and finally $F_{l k}\left(K, x_{k}\right)$ contains no point of the process, with probability exp$\theta\left(F_{l k}\left(K, x_{k}\right)\right)$. Equation (11) is obtained by randomization of the point $x_{k}$, followed by a randomization of the choice of metrics $d_{k}^{p}$ and $d_{l}^{p}$.

It is possible to replace the deterministic intensity $\theta(x)$ by a realization of a positive random function, replacing the Poisson point process by a Cox process [3]. In that case, we obtain Cox based random tessellations. Their corresponding moments $P(K)$ are deduced from equations $(9,11)$ by taking their expectation with respect to the random intensity.

\section{Extension to Johnson-Mehl and to Laguerre random tessellation}

The Johnson-Mehl tessellation [14] is obtained by combining germination (through a sequential intensity $\theta(t)$ ) and growth (with growth rate $\alpha(t)$ ). The usual model is based on constant (with respect to time) germination (with intensity $\theta$ ) and growth rate (with intensity $\alpha$ ). During the time sequence, germs falling inside growing crystals are deleted. Considering the sequence of Poisson germs $\left\{x_{k}, t_{k}\right\}$, we have:

$$
\mathrm{C}_{k}=\left\{x \in R^{n}, d\left(x,\left(x_{k}, t_{k}\right)\right)+\alpha\left(t_{k}\right) t_{k}<d\left(x,\left(x_{l}, t_{l}\right)\right)+\alpha\left(t_{l}\right) t_{l},\right.
$$

$\left.x_{k} \in \mathcal{P}, x_{l} \in \mathcal{P}, l \neq k\right\}$

Extensions of this model are obtained by means of a $L_{p}$ metric, instead of the Euclidean distance. We can also use a local metric in the process, to generate anisotropic growth:

$$
\mathrm{C}_{k}=\left\{x \in R^{n}, d_{k}\left(x,\left(x_{k}, t_{k}\right)\right)+\alpha\left(t_{k}\right) t_{k}<d_{l}\left(x,\left(x_{l}, t_{l}\right)\right)+\alpha\left(t_{l}\right) t_{l},\right.
$$
$\left.x_{k} \in \mathcal{P}, x_{l} \in \mathcal{P}, l \neq k\right\}$

The Laguerre tessellation $[9,10]$ is a generalization of the Voronoi tessellation, where to each Poisson point $x_{k}$ is attached a random radius $R_{k}$. The cell is now defined from the power $P\left(x, x_{k}\right)=d^{2}\left(x, x_{k}\right)-R_{k}^{2}$. We have:

$$
C_{k}=\left\{x \in R^{n}, P\left(x, x_{k}\right)<P\left(x, x_{l}\right), x_{k} \in \mathcal{P}, x_{l} \in \mathcal{P}, l \neq k\right\}
$$

Some germs $x_{l}$ generate empty cells, depending on the values of $R_{k}^{2}$ and on the distance to other germs. Cells are bounded by portions of hyperplanes. New random tessellations can be defined, based on the $L_{p}$ metric, replacing in equation (13) $P\left(x, x_{k}\right)$ by $d^{p}\left(x, x_{k}\right)-R_{k}^{p}$. In general, non planar cell separations will be generated.

An extension of the construction (8) to the local metric case is obtained if $P\left(x, x_{k}\right)$ and $P\left(x, x_{l}\right)$ are replaced by $d_{k}^{p}\left(x, x_{k}\right)-R_{k}^{p}$ and $d_{l}^{p}\left(x, x_{k}\right)-R_{l}^{p}$ in equation (13). 


\section{Random tessellations and Boolean random functions}

The previous constructions can be obtained as characteristics of some Boolean random functions, re-interpreting the definition of $C_{k}$ in terms of distance function.

We will attach to every Poisson point $x_{k}$ a primary random function $Z_{k}^{\prime}(x)$ defined according to the distance used in various definitions $(4,8,4,4,13)$. For instance, for the standard Voronoi model, the primary function is an increasing paraboloid of revolution, while for the extension to the local Euclidean metric, it is an increasing paraboloid with general ellipsoidal sections in $R^{n}$. For the Johnson-Mehl model, primary functions are cones (with ellipsoidal section in the local case for the Euclidean metric); for germ $\left\{x_{k}, t_{k}\right\}$, the primary function is translated upward by addition of the constant $\alpha\left(t_{k}\right) t_{k}$. Models based on the $L_{p}$ metric make use of functions defined in $R^{n}$ by $Z_{k}^{\prime}(x)=\sum_{i=1}^{i=n} a_{k i}\left|x_{i}^{\prime}\right|^{p}$. The distance function associated to a model built from Poisson germs is given by

$$
Z(x)=\wedge_{k} Z_{k}^{\prime}\left(x-x_{k}\right)
$$

By definition the random function $Z(x)$ is an Infimum Boolean random function [6]. For the Johnson-Mehl model, the primary function becomes $Z_{k}^{\prime}\left(x-x_{k}\right)+$ $\alpha\left(t_{k}\right) t_{k}$. For the Laguerre tessellation model, it becomes $Z_{k}^{\prime}\left(x-x_{k}\right)-R_{k}^{p}$.

Sections of primary functions at level $z$ are balls defined by the corresponding metric. Define

$$
B_{k}^{\prime}(z)=\left\{x, Z_{k}^{\prime}(x)<z\right\}
$$

From equation (14) we have

$$
B(z)=\{x, Z(x)<z\}=\cup_{x_{k}} B_{k}^{\prime}(z)_{x_{k}}
$$

By construction equation (15) $B(z)$ is a Boolean random set with convex primary grains $B_{k}^{\prime}(z)$. Consider a compact set $K$ and the infimum $Z_{\wedge}(K)=$ $\wedge_{y \in K}\{Z(y)\}$. We have

$$
P\left\{Z_{\wedge}(K) \geq z\right\}=\exp -\left\{E\left(\theta\left(B_{k}^{\prime}(z) \oplus \check{K}\right)\right)\right\}
$$

and for the stationary case

$$
P\left\{Z_{\wedge}(K) \geq z\right\}=\exp -\left\{\theta E\left(\mu_{n}\left(B_{k}^{\prime}(z) \oplus \check{K}\right)\right)\right\}
$$

For the simulation of random tessellations, we just need to simulate realizations of the Boolean random function with primary functions $Z_{k}^{\prime}$ corresponding to the model. The boundaries of the tessellation are provided by the crest lines of the random functions, obtained by the watershed of the random function using as markers the Poisson points. By construction of the Boolean random functions, the location of crest lines, and therefore the boundaries of the classes of the resulting tessellation are invariant by a non decreasing transformation $\Phi$ (anamorphosis) of the values of $Z_{k}^{\prime}(x)$ (for instance using $Z_{k}^{\prime p}(x)$ instead of $\left.Z_{k}^{\prime}(x)\right)$, that is compatible with the order relationship, namely such that $z_{1}<z_{2}$ 
implies $\Phi\left(z_{1}\right)<\Phi\left(z_{2}\right)$. An alternative extraction of classes is given by their labels $C_{k}$. Starting from the simulation, and from the germs $x_{k}$, we generate in each point $x$ a set of labels $L(x)$ :

$$
L(x)=\left\{k, Z(x)=Z_{k}^{\prime}\left(x-x_{k}\right)\right\}
$$

Points $x$ with the single label $k$ generate the interior of cell $C_{k}$. Points with two labels $k$ and $l$ are on the boundaries between cells $C_{k}$ and $C_{l}$. In $R^{3}$, points with three labels are on the edges of the tessellation, and points with four labels are its vertices.

This is illustrated in Figure 1 by a simulation in $R$, where a non connected class $C_{k}$ is generated by the point $x_{k}$. This is just obtained by application of equation (18), the distance to $x_{k}$ of points located in the left part of $C_{k}$ of the figure being shorter than the distance to other germs.

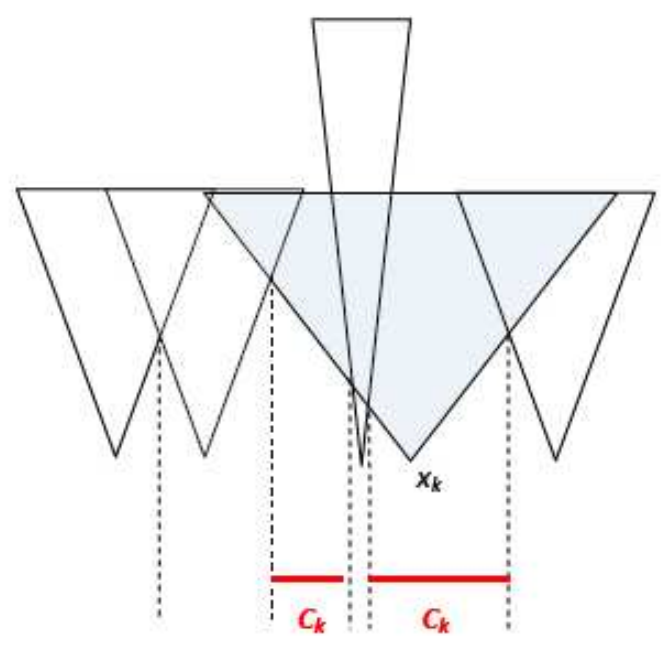

Figure 1 : Example of simulation of a random tessellation in $R$ by means of a Boolean random function with different primary functions. The class $C_{k}$, generated by germ $x_{k}$, is not connected.

In Figure 2 is shown a realization of a Boolean random function BRF where the primary functions are doublets of elliptical cones with two orthogonal directions (the two vertical cones being obtained by a horizontal translation, and the two horizontal cones by a vertical translation) and the same minima. Figure 3 shows the corresponding local $L_{2}$ Voronoï random tessellation obtained from the watershed of the BRF. A simulation of a Boolean random function with vertial and horizontal elliptical cones having different minima is given in Figure 4. Its watershed in Figure 5 generates a realization of a local $L_{2}$ Johnson-Mehl random tessellation. 


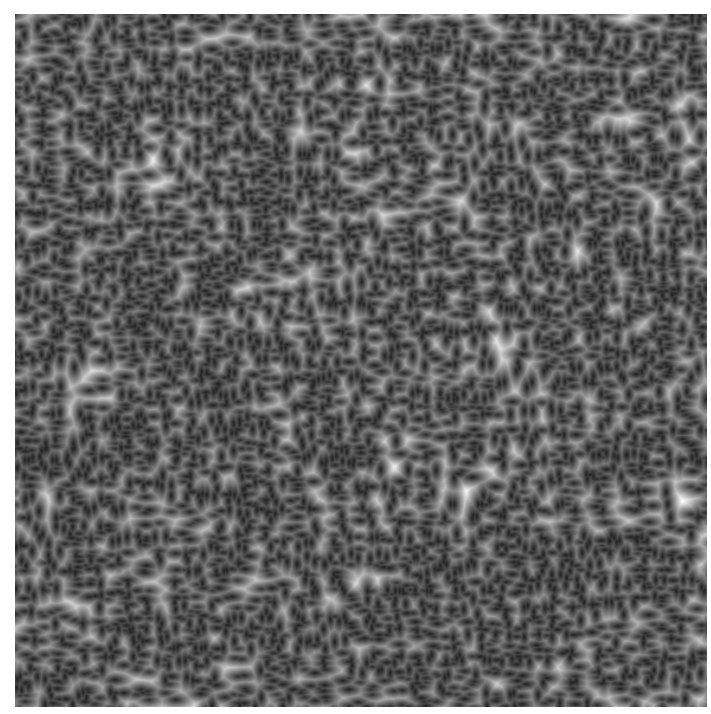

Figure 2: Example of simulation of a Boolean random function with elliptical cones doublets in two orthogonal directions (image $800 \times 800$ ).

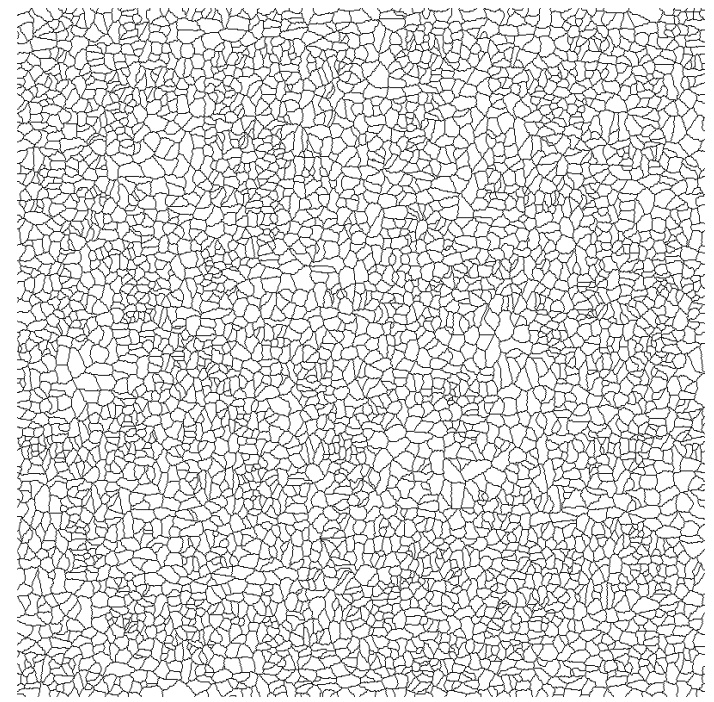

Figure 3: Local $L_{2}$ Voronoï random tessellation generated by the realization of the Boolean random function of Figure 2 (image 800 x 800). The classes of the tessellation are obtained as the attraction zones of the minima of the BRF 

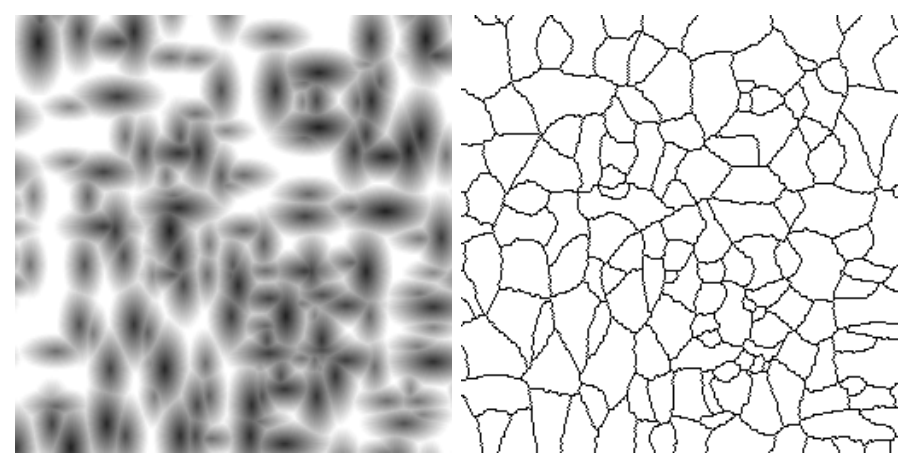

Figure 4 (left) : Example of simulation of a Boolean random function with elliptical cones in two orthogonal directions. The primary functions start from different values (image $256 \times 256$ ).

Figure 5 (right): Local $L_{2}$ Johnson-Mehl random tessellation generated by the realization of the Boolean random function of Figure 4 (image 256 x 256). The

classes of the tessellation are obtained from the watershed of the BRF.

More general random tessellations can be generated by the same process, starting from Boolean random functions with any primary random function $Z^{\prime}(x)$. We consider that the realization $k$ of $Z^{\prime}(x)$ is characterized by some multivariate distribution $\varphi(k)$, and owns simply connected compact sections $B_{k}^{\prime}(z)$, such that $B_{k}^{\prime}\left(z_{1}\right) \subset B_{k}^{\prime}\left(z_{2}\right)$ for $z_{2}>z_{1}$. We consider primary random functions reaching their minimum $Z^{\prime}(0)$ for $x=0$. We associate to $Z_{k}^{\prime}(x)$ the floor set $A_{k}^{\prime}$ defined by

$$
A_{k}^{\prime}=\left\{x, Z_{k}^{\prime}(x)=Z_{k}^{\prime}(0)\right\}
$$

In all previous situations we had $A^{\prime}=\{O\}$. If for any pair of Poisson points $\left(x_{k}, x_{l}\right)$ we have $A_{k x_{k}}^{\prime} \cap A_{l x_{l}}^{\prime}=\varnothing$, we can define the class $C_{k}$ of the random tessellation, generated by the germ $x_{k}$ and the primary random function $Z^{\prime}(x)$ by:

$$
C_{k}=\left\{x \in R^{n}, Z_{k}^{\prime}\left(x-x_{k}\right)<Z_{l}^{\prime}\left(x-x_{l}\right), x_{k} \in \mathcal{P}, x_{l} \in \mathcal{P}, l \neq k\right\}
$$

This construction of classes associated to germs works when $A^{\prime}=\{O\}$. In $R^{2}$ it can also be applied when the floor set is made of parallel segments, while segments with two different orientations may overlap. In $R^{3}$, local anisotropy can be obtained by the Euclidean distance function to segments with different orientations, or even by Poisson lines $[12,5]$ with an infinite length. In that case, point Poisson germs are replaced by segment germs or by lines. The generation of classes in simulations can be made by means of the previous procedure involving labels $L(x)$.

Generalizing the previous case of local metrics, we call $F_{l k}(K, y)$ the flower of $K$ with center $y$, and primary functions $Z_{k}^{\prime}(x)$ and $Z_{l}^{\prime}(x)$. We have

$$
F_{l k}(K, y)=\cup_{x \in K} B_{l}^{\prime}\left(Z_{k}^{\prime}(x-y)\right)_{x}
$$

The previous results are extended as follows. 
Theorem 3. Consider a random tessellation of space defined from the Poisson point process with intensity $\theta(x)$ and the primary random function $Z^{\prime}(x)$ generating the flower defined by equation (21). The probability $P(K)=P\left(K \subset C_{k}\right)$ is given by

$$
P(K)=\int_{R^{n}} \varphi(l) d l \varphi(k) d k \theta(d y) \exp -\theta\left(F_{l k}(K, y)\right)
$$

In the stationary case, for a constant intensity $\theta$, equation (11) becomes

$$
P(K)=\theta \int_{R^{n}} \varphi(l) d l \varphi(k) d k \exp -\theta \mu_{n}\left(F_{l k}(K, y)\right) d y
$$

Proof. Conditionally to the primary functions $Z_{k}^{\prime}(x)$ and $Z_{l}^{\prime}(x)$, and to the location of a Poisson point $x_{k}$, we have $K \subset C_{k} \Longleftrightarrow \forall x \in K, \forall l \neq k$, $Z_{k}^{\prime}\left(x-x_{k}\right)<Z_{l}^{\prime}\left(x-x_{l}\right)$. Therefore, for every point $x$ of $K$, the set $B_{l}^{\prime}\left(Z_{k}^{\prime}\left(x, x_{k}\right)\right)_{x}$ contains no point of the process, and finally $F_{l k}\left(K, x_{k}\right)$ contains no point of the process, with probability $\exp -\theta\left(F_{l k}\left(K, x_{k}\right)\right)$. Equation (11) is obtained by randomization of the point $x_{k}$, followed by a randomization of the choice of the primary functions $Z_{k}^{\prime}(x)$ and $Z_{l}^{\prime}(x)$.

As before, replacing the deterministic intensity $\theta(x)$ by a realization of a positive random function $\Theta$, we obtain Cox based random tessellations with corresponding moments $P(K)$ deduced from equation (22) by taking its expectation with respect to the random intensity $\Theta$.

\section{Some indications on model identification}

For practical applications, the choice of a proper model has to be done from available information, usually 2D or 3D images of the microstructures. Several criteria can be used to select a representative model: the boundaries of cells can be fit to polynomials of degree $p$, given the order of the $L_{p}$ metric. In a work on metallic grains fom EBSD images [1], pertinent information on local metrics is extracted from the inertia matrix of the grains. Finally, use should be made of the functional $P(K)$ computed from the equations or estimated from measurements on real or simulated microstructure. As a consequence of the presence of non connected classes generated by the models, the measurements on images should ne restricted to connected compact sets $K$, directly obtained by erosion of the complementary set of the boundaries of classes by $K$.

\section{Conclusion}

Random tessellations models involving Poisson germs were revised and generalized by the use of local metrics attached to the germs. These models are related to particular Boolean random functions with particular primary functions generated by the metrics. Using other primary functions extends the type of random 
tessellations that can be simulated, and gives more flexibility to model complex real microstructures. Further generalizations of random tessellations can be obtained on Riemannian manifolds, equipped with a Riemannian metric, zones of influence of random points being generated by means of a geodesic distance, as already studied on the sphere [13]. Most results on the present study can be extended to this situation. In $R^{n}$, the $L_{p}$ metrics can also be replaced by a geodesic distance, giving access to more general models.

\section{References}

1. Altendorf H., Latourte F., Saintoyant L., Jeulin D., Faessel M. (2013) Analysis and Stochastic Modeling of the Microstructures of Steel P91/P92 for Fracturation Studies at Welding Joints, submitted to ECS 11, Kaiserslautern, July 2013.

2. Calka P. (2013) Asymptotic methods for random tessellations, chapter 6 in: Stochastic Geometry, Spatial Statistics and Random Fields, E. Spodarev (ed.), Lecture Notes in Mathematics, Vol. 2068, Springer Verlag.

3. Cox D. R. (1955) Some statistical methods connected with series of events, Journal of the Royal Statistical Society. Series B, Vol. 17, 129-164.

4. Gilbert E.N. (1962) Random subdivisions of space into crystals, The Annals of mathematical statistics, Vol. 33, 958-972.

5. Jeulin D. (1991) Modèles de Fonctions Aléatoires multivariables. Sci. Terre; 30: 225-256.

6. Jeulin D., Modèles Morphologiques de Structures Aléatoires et de Changement d'Echelle, Thèse de Doctorat d'Etat ès Sciences Physiques, Université of Caen, 1991.

7. T., S. Forest, Galliet I., Mounoury V., Jeulin D., Determination of the size of the representative volume element for random composites: statistical and numerical approach, Int. J. Sol. Str., Vol. 40 (2003) 3647-3679.

8. Labelle F., Shewchuk J.R. (2003) Anisotropic Voronoi Diagrams and GuaranteedQuality Anisotropic Mesh Generation, Proceedings of the nineteenth annual symposium on Computational geometry, San Diego, 191-200.

9. Lautensack C., Zuyev S. (2008) Random Laguerre Tessellations, Advances in applied probability, Vol. 40, 630-650.

10. Lautensack C. (2008) Fitting three-dimensional Laguerre tessellations to foam structures, Journal of Applied Statistics, Vol. 35 (9), 985-995.

11. Matheron G. (1969) Introduction à la théorie des ensembles aléatoires, Cahiers du Centre de Morphologie Mathématique, fascicule 4.

12. Matheron G. (1975) Random sets and Integral Geometry, Wiley.

13. Miles R.E. (1971) Random Points, Sets and Tessellations on the Surface of a Sphere, Sankhyā : The Indian Journal of Statistics, Series A, 1971, 145-174.

14. Møller J. (1992) Random Johnson-Mehl tessellations, Advances in applied probability, Vol. 24, 814-844.

15. Scheike Th. H. (1994) Anisotropic growth of Voronoi Cells, Advances in applied probability, Vol. 26, 43-53.

16. Serra J., Image analysis and Mathematical Morphology, Academic Press, London, 1982.

17. Stoyan, D., W.S. Kendall, J. Mecke (1987) Stochastic Geometry and its Applications (J. Wiley, New York). 